L. Kowalzick

D. Iliev

\section{Erfahrungen mit der Anwendung von polychromatischem polarisierten Licht auf Spalthautentnahmestellen}

\author{
Experiences with the Administration of Polychromatic Polarized Light \\ on Split Skin Graft Donor Sites
}

\section{Zusammenfassung}

Seit kurzem wird die Bestrahlungsbehandlung von Hautwunden mit polarisiertem Licht zum Zwecke der Förderung der Wundheilung propagiert. Ein entsprechendes Bestrahlungssystem (Q.Light $200^{\circledR} \mathrm{NT}$ ) ist als Medizinprodukt der Klasse II zertifiziert. Wir wendeten polarisiertes, nichtkohärentes, polychromatisches weißes Licht (385-780 nm) in einer Dosierung von ca. $56 \mathrm{~J} / \mathrm{cm}^{2}$ einmal tgl. über 3 Wochen zur Bestrahlung von 0,3 mm tiefen Spalthautentnahmewunden vom Oberschenkel bei 5 Patienten an. Die Wundflächen waren nach Spalthautentnahme durchschnittlich $125 \mathrm{~cm}^{2}$ groß. Nach ein-, zwei- und dreiwöchiger Behandlung waren sie zu 36\%, 63\% und 83\% reepithelialisiert. Abgesehen von einem Patienten, bei dem sich am Ende der Behandlungsserie einzelne kleine Blasen im Bereich der reepithelialisierten Haut auf der belichteten Seite zeigten, traten keine unerwünschten Wirkungen auf. Unsere Beobachtungen sprechen dafür, dass unter Belichtung mit tgl. $56 \mathrm{~J} / \mathrm{cm}^{2}$ polarisiertem weißen Licht die Wundheilung von $0,3 \mathrm{~mm}$ tiefen Spalthautentnahmestellen gut voranschreitet, diese aber hierdurch nicht wesentlich beschleunigt wird.

\section{Patienten und Methoden}

Behandelt wurden 5 Patienten mit venös bedingten, konservativ therapierefraktären, ausgedehnten Ulcera crurum, die zur operativen Deckung nach Wundkonditionierung die Entnahme ausgedehnter Spalthauttransplantate bedurften. Es handelte sich um 3 weibliche und 2 männliche Patienten im Alter von durchschnittlich 68 Jahren (53-83 Jahre). Eine Patientin litt zusätzlich an ei-

\section{Abstract}

Recently, treatment with polarized light was advocated for enhancing skin wound healing. A corresponding treatment device (Q.Light ${ }^{\circledR} 200 \mathrm{NT}$ ) was certified as class II medicine product. We administered polarized, non coherent, polychromatic white light $(385-780 \mathrm{~nm})$ in a dose of approx. $56 \mathrm{~J} / \mathrm{cm}^{2}$ once daily for 3 weeks in 5 Patients with $0.3 \mathrm{~mm}$ deep split skin graft donor site wounds of the upper leg. The average wound surface at time of grafting measured $125 \mathrm{~cm}^{2}$. After one, two and three weeks of treatment $36 \%, 63 \%$ and $83 \%$ of the wound surface had reepithelised, respectively. No unwanted side effects occurred except some small blisters in reepithelised skin on the irradiated side in one patient near the end of treatment. Our observations indicate that during daily administrations with $56 \mathrm{~J} / \mathrm{cm}^{2}$ polarized white light wound healing of $0.3 \mathrm{~mm}$ deep split skin graft donor sites proceeds well, but is not markedly enhanced. nen Diabetes mellitus, eine andere an einer arteriellen Hypertonie mit koronarer Herzkrankheit.

Die Spalthaut wurde in standardisierter Weise in TumeszenzLokalanästhesie mittels Elektrodermatom in einer Schichtdicke von $0,3 \mathrm{~mm}$ vom Oberschenkel entnommen. Die rechteckigen Entnahmewunden massen durchschnittlich $125 \mathrm{~cm}^{2}$ $\left(61-321 \mathrm{~cm}^{2}\right)$. Erstmals 24 Stunden nach der Spalthautentnah- 


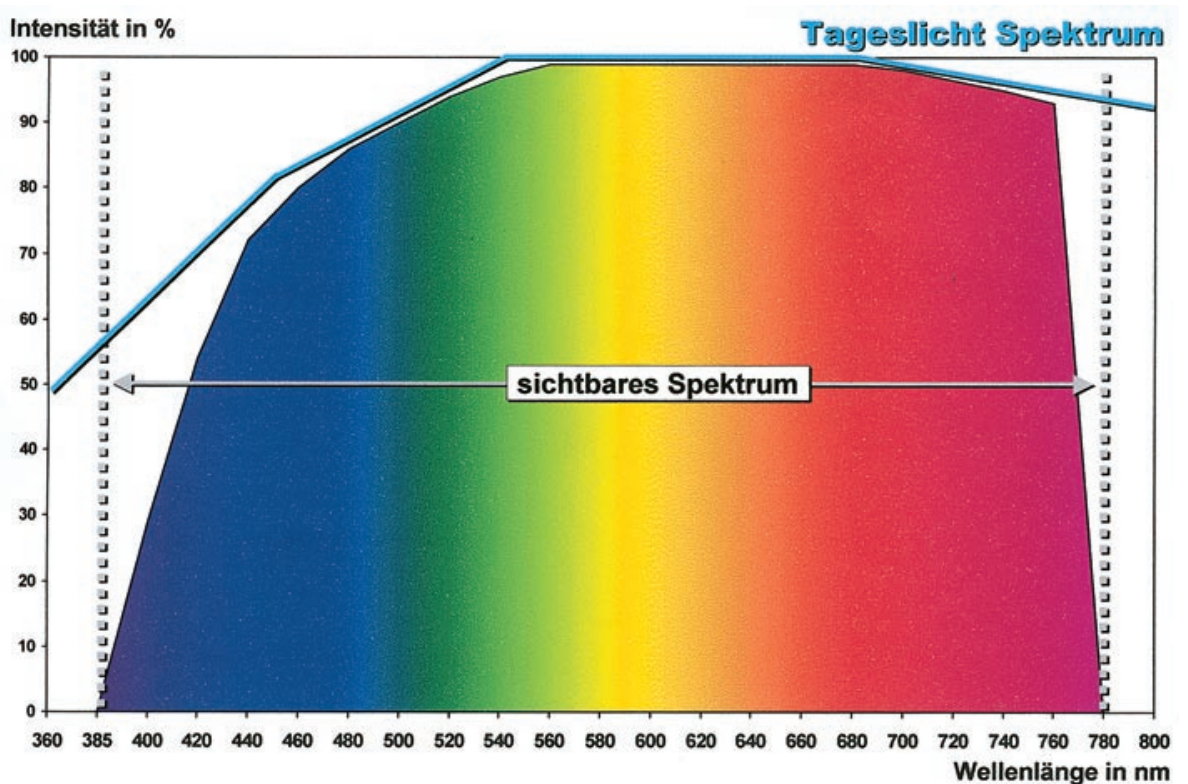

Abb. 1 Relatives Emissions-Spektrum des Q.Light ${ }^{\circledR} 200$ NT Belichtungsquelle im Vergleich zum solaren Spektrum nach Herstellerangaben.

me, und dann täglich über 3 Wochen, erfolgte ein Verbandswechsel mit Pasta zinci molle und sterilen Metalline ${ }^{\circledR}$ Wundauflagen. Nach vorsichtiger Entfernung der Wundauflage einschließlich Pastenresten und vorsichtiger Wundreinigung erfolgte dann im Rahmen eines individuellen Heilversuches täglich die Belichtung der Wunde, anschliessend wurde die Wunde wieder verbunden.

Die Belichtung erfolgte mit dem Q.Light ${ }^{\circledR} 200$ NT-Lichttherapiegerät der Firma biotechnologie \& photomedizin Schweiz AG (CH-9404 Rorschacher Berg). Dieses Gerät, ein Halogenhochdruckstrahler emittiert sichtbares, nicht-kohärentes, polychromatisches weißes Licht mit Wellenlängen von 385 bis $780 \mathrm{~nm}$ ohne sonstige ultraviolette und infrarote Anteile. Die relativen spektralen Bestrahlungsstärken entsprechen weitgehend denen des sichtbaren Sonnenlichts (Abb.1). Das Gerät hat bei $40 \mathrm{~cm} \mathrm{Ab-}$ stand eine Bestrahlungsstärke von $40 \mathrm{~mW} / \mathrm{cm}^{2}$ entsprechend einer Dosisleistung von $2,4 \mathrm{~J} / \mathrm{cm}^{2}$ pro Minute. Die Lichtstärke beträgt 2500 Lux. In diesem Abstand können bis zu $40 \mathrm{~cm}$ durchmessende Felder belichtet werden. Das Gerät ist als Medizinprodukt der Klasse II zertifiziert und trägt das CE-Zeichen gemäss Anhang VI der Richtlinie für Medizinprodukte 93/42/EWG. In den Lichtstrahlengang wurde der Q.Light ${ }^{\circledR}$ Polarisationsfilter eingeführt. Wir bestrahlten in einem Abstand von $30 \mathrm{~cm}$ für tgl. 12 Minuten, entsprechend einer Dosis von jeweils $56 \mathrm{~J} / \mathrm{cm}^{2}$ über insgesamt 3 Wochen.

\section{Ergebnisse}

Nach ein-, zwei- und dreiwöchiger Behandlung waren die Spalthautentnahmewunden zu 36\%,63\% und 83\% reepithelialisiert (Abb. 2, Tab.1). Ein Beispiel für einen klinischen Verlauf zeigt Abb. 3. Bei diesem Patienten wurde eine ansonsten gleich versorgte Wunde am kontralateralen Oberschenkel nicht belichtet. Abgesehen von einem Patienten, bei dem sich am Ende der Behandlungsserie einzelne kleine Blasen im Bereich der reepithelialisierten Haut auf der belichteten Seite zeigten (Abb. 4), traten keine unerwünschten Nebenwirkungen auf.

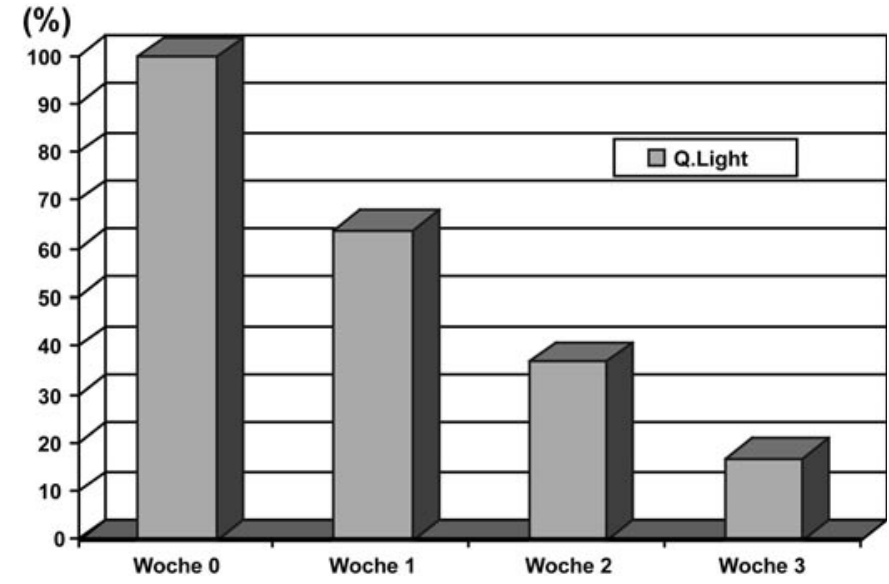

Abb. 2 Durchschnittliche Wundfläche nach 0,3 mm tiefer Spalthautentnahme am Oberschenkel in Prozent des Ausgangswertes bei $5 \mathrm{~Pa}$ tienten vor und nach ein-, zwei- und dreiwöchiger Behandlung mit polarisiertem weißen Licht (Q.Light ${ }^{\circledR} 200$ NT).

\section{Diskussion}

Trotz ermutigender Resultate in vitro und im Tiermodell waren die Resultate klinischer Untersuchungen mit Low-level-Lasertherapie zur Förderung der Wundheilung enttäuschend [4]. In den 1980er Jahren erschienen einige Arbeiten über die Anwendung von polarisiertem Licht bei der Behandlung chronischer Hautwunden $[8,9]$. Seit Kurzem wird erneut über Erfolge bei der Anwendung von polarisiertem Licht zur Förderung der Wundheilung bei Brandwunden, venösen und Decubital-Ulcera berichtet $[2,5,6]$. Als mögliche Wirkungsmechanismen werden u.a. Einflüsse auf Fibroblastenproliferation, Makrophagen- und Lymphozytenfunktionen sowie Zytokin- und Wachstumsfaktoren-Produktion diskutiert $[1,3,10,11]$.

Stäcker [8] behandelte über 200 chronische Hautwunden unterschiedlichster Art, u.a. Decubitalläsionen, venöse und arteriell bedingte Ulcera, diabetische Ulcera und septische chirurgische Wunden mit polarisiertem Licht und Infrarot mit einer Wellen- 

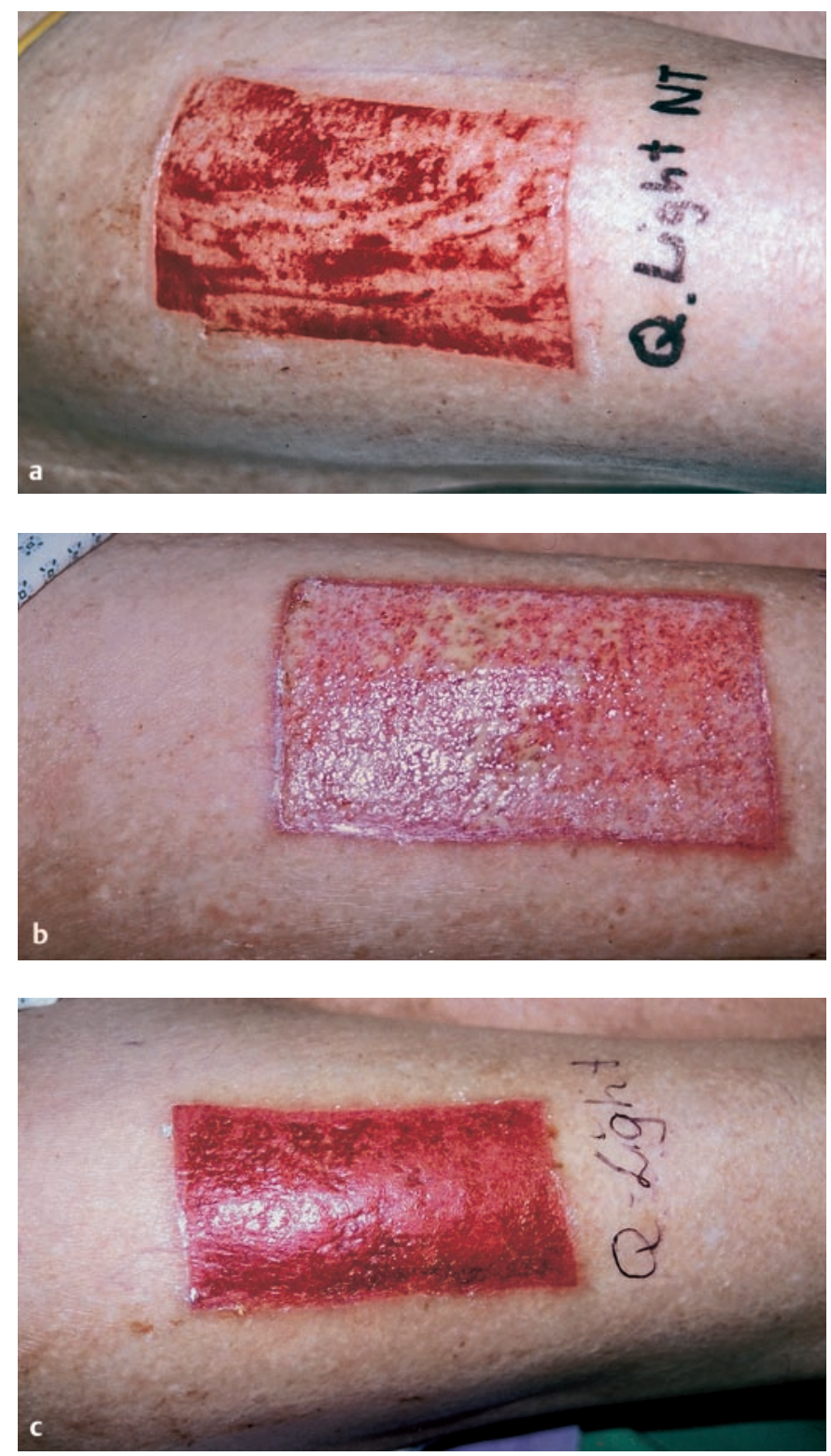

Abb. 3 Wunden nach 0,3 $\mathrm{mm}$ tiefer Spalthautentnahme an beiden Oberschenkeln einer 83-jährigen Patientin jeweils am 1. postoperativen Tag und nach ein- und dreiwöchiger Behandlung mit polarisiertem

länge von 500 bis $3000 \mathrm{~nm}$ in einer tgl. Dosis von $4,89 \mathrm{~J} / \mathrm{cm}^{2}$ bei einem Bestrahlungsabstand von $20 \mathrm{~cm}$ über mehrere Wochen, z.T. kombiniert mit operativen Deckungsverfahren und berichtet kasuistisch über gute Ergebnisse. Mit dem gleichen Schema behandelte Stegmann [9], der das Spektrum der Belichtungsquelle mit 400 bis $3000 \mathrm{~nm}$ angibt, 52 Patienten mit venösen Ulcera crurum, wobei bei $26 \%$ eine Abheilung und bei 13 eine wesentliche Besserung eingetreten sei.

Monstrey et al. [5] behandelten 22 Patienten mit tiefen dermalen Brandwunden mit polarisierten Licht und Infrarot von 400-2000 nm Wellenlänge mit einer Bestrahlungsstärke von $40 \mathrm{~mW} / \mathrm{cm}^{2}$ und einer tgl. Dosis von $14,4 \mathrm{~J} / \mathrm{cm}^{2}$ bei einem Bestrahlungsabstand von $10 \mathrm{~cm}$ bis zur Abheilung nach durchschnittlich 3,2 Wochen. Diese konservative Therapie hätte, verglichen mit den Erwartungen einer Expertengruppe zu einer signifikant kürzeren Wundheilungszeit geführt. Medenica et al. be-
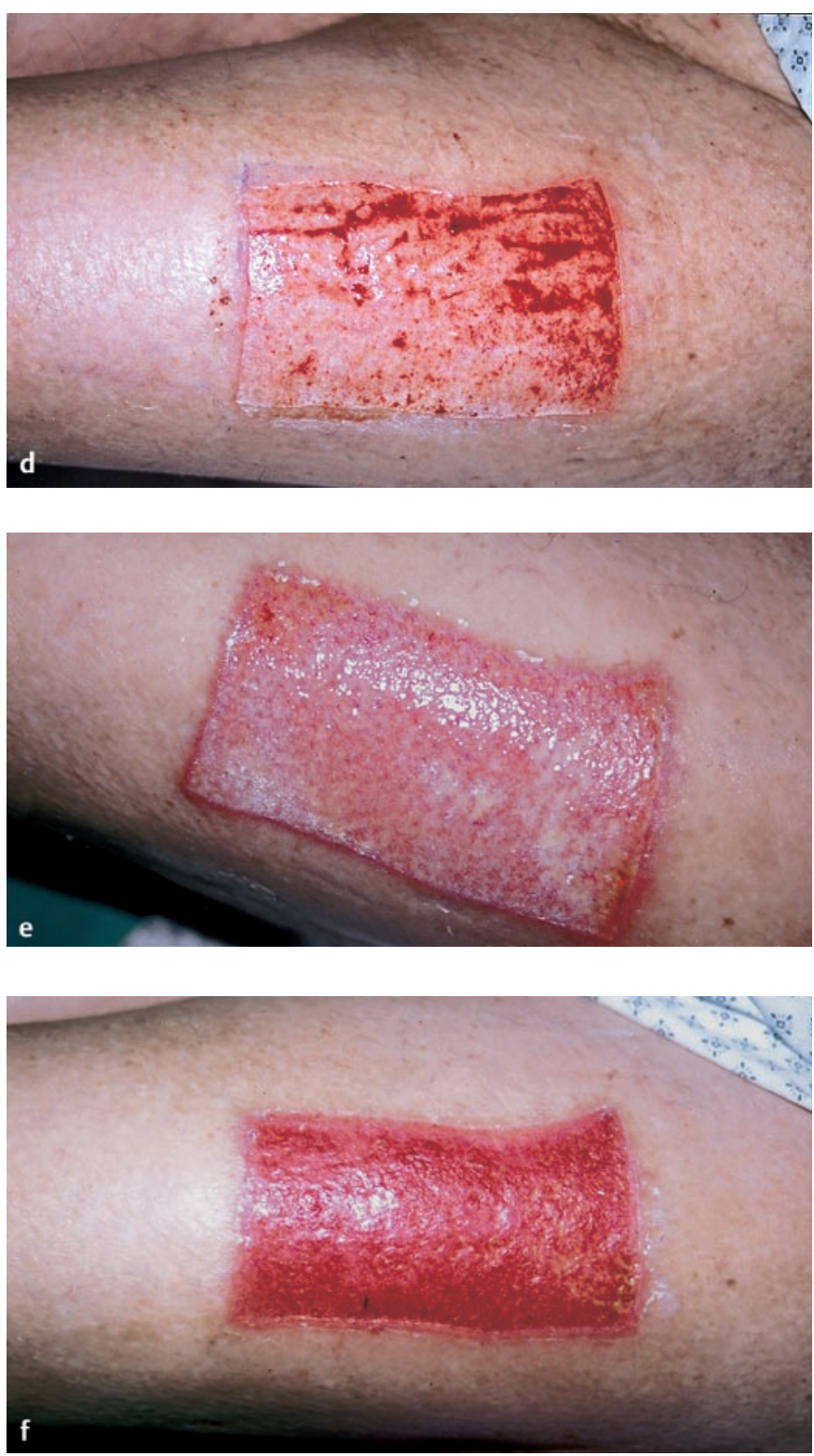

weißen Licht (Q.Light ${ }^{\circledR} 200$ NT) (a-c) bzw. ausschließlich täglichen Verbandswechseln (Kontrolle) (d-f).

handelten 2003 in einer prospektiven unkontrollierten Fallstudie [6] 25 Patienten mit zusammen 73 venösen Ulcera crurum mit polarisiertem, nichtkohärenten polychromatischen Licht und Infrarot aus einer Lichtquelle desselben Herstellers, wobei die Wellenlänge mit 480-3400 nm angegeben wird, und einer Dosis von täglich 19,3 J/ $\mathrm{cm}^{2}$ bei einem Bestrahlungsabstand von $10 \mathrm{~cm}$ über vier Wochen. Die Zahl der noch offenen Ulcera und die Wundflächen nahmen jeweils signifikant über den Behandlungszeitraum ab. Mit einer Lichtquelle des selben Herstellers wurde bei 55 Patienten mit beidseitigen Decubitalulcera jeweils eines über 2 Wochen werktäglich für $5 \mathrm{~min}$ mit $20 \mathrm{~J} / \mathrm{cm}^{2}$ polarisiertem Licht (und Infrarot?) behandelt [2]. Die Wundoberfläche nahm während dieses Behandlungszeitraumes auf der belichteten Seite von durchschnittlich 2,84 auf $2,26 \mathrm{~cm}^{2}$ und auf der unbestrahlten Seite von 2,10 auf $2,04 \mathrm{~cm}^{2}$ ab, wobei während der ersten Behandlungswoche die Abnahme der Wundfläche nur auf der belichteten Seite signifikant war. Über den gesamten 
Tab. 1 Patienten- und Wundcharakteristika sowie Reepithelialisierungsgrad in Prozent des Ausgangswertes von mit polarisiertem weißen Licht behandelten 0,3 mm tiefen Spalthautentnahmewunden vom Oberschenkel

\begin{tabular}{llllllr}
\hline Patient & Alter & Geschl. & $\begin{array}{l}\text { Wundfläche } \\
\text { vor Therapie }\end{array}$ & $\begin{array}{l}\text { abgeheilte Wundfläche } \\
\text { Woche 1 (\%) }\end{array}$ & $\begin{array}{l}\text { abgeheilte Wundfläche } \\
\text { Woche 2 (\%) }\end{array}$ & $\begin{array}{l}\text { abgeheilte Wundfläche } \\
\text { Woche 3 (\%) }\end{array}$ \\
\hline C. S. & 69 & W & $81,2 \mathrm{~cm}^{2}$ & 30 & 50 & 90 \\
\hline A. R. & 73 & $\mathrm{~m}$ & $320,5 \mathrm{~cm}^{2}$ & 50 & 95 & 100 \\
\hline H. U. & 62 & $\mathrm{~m}$ & $131,3 \mathrm{~cm}^{2}$ & 30 & 50 & 70 \\
\hline G. S. & 53 & W & $121,0 \mathrm{~cm}^{2}$ & 60 & 60 & 95 \\
\hline M. B. & 83 & W & $66,0 \mathrm{~cm}^{2}$ & 10 & 60 & 60 \\
\hline
\end{tabular}

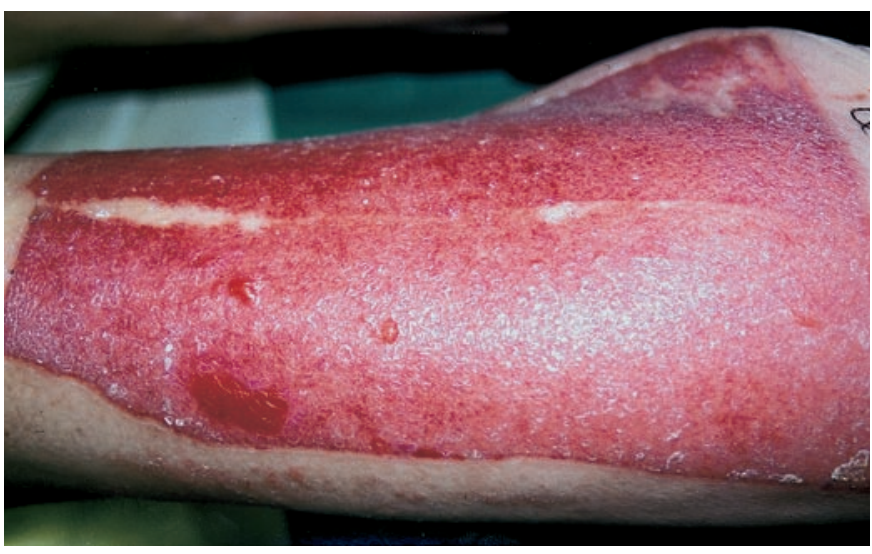

Abb. 4 Blasenbildung im Bereich 6 Tage vorher dort bereits vollständig reepithelialisierter Spalthautentnahmewunde nach 29. Belichtung mit $56 \mathrm{~J} / \mathrm{cm}^{2}$ polarisierten weißen Lichtes bei 73-jährigem Patienten.

Zeitraum war die Exsudation auf der bestrahlten Seite signifikant geringer und das Verhältnis der rosa/weiß Farbwerte signifikant höher als beim unbehandelten Ulcus.

In einer weiteren Untersuchung wurden Spalthautentnahmestellen bei 10 Patienten mit polarisierten, inkohärenten Licht tgl. für 6 Minuten behandelt. Jeweils die Hälfte der Wundfläche wurde hierbei mittels Aluminiumfolie abgedeckt. Hierbei heilten die belichteten Wundanteile durchschnittlich 2 Tage früher ab; der Unterschied war jedoch statistisch nicht signifikant [7]. In einer parallelen Untersuchung im Tiermodell wurden bei 7 Schweinen $0,6 \mathrm{~mm}$ tiefe Spalthautentnahmen von $8 \times 4 \mathrm{~cm}$ durchgeführt, und die Wunden alle 2 Tage in gleicher Weise belichtet, wobei die histologisch verifizierte Abheilungszeit bestrahlter Wunden statistisch signifikant um einen Tag verkürzt wurde ( 7 vs. 8 Tage). Die verwendete Wellenlänge und die applizierte Dosis wurde allerdings nicht mitgeteilt.

Die Beurteilung der verschiedenen Untersuchungen wird durch die teilweise unpräzisen Angaben über die verwendeten Lichtquellen und speziell über die effektive Dosis im Behandlungsfeld erschwert. Die verschiedenen Untersucher behandelten auch ganz unterschiedliche chronische Wunden und verwendeten unterschiedliche Spektren und Dosen. Fasst man auch unter diesen
Aspekten die Ergebnisse zusammen, so erscheint es zwar möglich, dass eine geringe Beschleunigung der Wundheilung durch die Belichtung mit polarisiertem nichtkohärenten Licht erreicht werden kann; zum Nachweis eines signifikanten Behandlungseffekts wären aber prospektive, kontrollierte und randomisierte klinische Studien an großen Kollektiven zu fordern. Eine auch nur geringe Senkung der Dauer bis zur Abheilung einer chronischen Wunde um einen oder wenige Tage könnte auch unter dem Aspekt der neuen Entgeltsysteme in der stationären Krankenversorgung Bedeutung erlangen.

\section{Literatur}

${ }^{1}$ Fenyö M, Mandl J, Falus A. Opposite effect of lineary polarized light on biosynthesis of interleukin- 6 in a human B lymphoid cell line and peripheral human monocytes. Cell Biol Internl 2002; 26: 265-269

2 Iordanou P, Baltopoulos G, Giannakopoulou M, Bellou P, Ktenas E. Effect of polarized light in the healing of pressure ulcers. Int J Nurs Pract 2002; 8: $49-55$

${ }^{3}$ Kubosova T, Horváth M, Kocsis K, Fenyö M. Effect of visible light on some cellular and immune parameters. Immunol Cell Biol 1995; 73: $239-244$

${ }^{4}$ Lucas C, Criens-Poublon LJ, Cockrell CT, de Haan RJ. Wound healing in cell studies and animal model experiments by low level laser therapy; were clinical studies justified? a systematic review. Laser Med Sci 2002; 17: $110-134$

${ }^{5}$ Medenica L, Lens M. The use of polarised polychromatic non-coherent light alone as a therapy for venous leg ulceration. J Wound Care 2003; 12: $37-40$

${ }^{6}$ Monstrey S, Hoeksema H, Saelens H, Depuydt K, Hamdi M, van Landuyt K, Blondeel P. A conservative approach for deep dermal burn wounds using polarised-light therapy. Brit J Plast Surg 2002; 55: $420-426$

${ }^{7}$ Schuhmann K, Ghofrani A, Höller D, Messmer BJ. Klinische und tierexperimentelle Untersuchungen zur Wirkung von polarisiertem, inkohärentem Licht an Spalthautentnahmestellen. Zentralbl Chir 1998; 123: 606

${ }^{8}$ Stäcker AD. Förderung der Wundheilung durch Bestrahlung mit polarisiertem Licht. Klinische Erfahrungen mit Evolite-Behandlungen. Med Welt 1986; 37: 1419-1423

${ }^{9}$ Stegmann W. Behandlung des Ulcus cruris mit polarisiertem Licht. Phlebol Proktol 1985; 14: 96 - 97

10 Vinck EM, Cagnie BJ, Cornelissen MJ, Declercq HA, Cambier DC. Increased fibroblast proliferation induced by light emitting diode and low power laser irradiation. Laser Med Sci 2003; 18: 95 - 99

11 Young S, Bolton P, Dyson M, Harvey W, Diamantopoulos C. Macrophage responsiveness to light therapy. Laser Surg Med 1989; 9: 497-505 